\title{
Swing-Up Control of the Acrobot: An Impulse-Momentum Approach
}

\author{
Rouhollah Jafari, Frank B. Mathis and Ranjan Mukherjee
}

\begin{abstract}
An impulse-momentum approach is proposed for swing-up control of the acrobot. The algorithm is based on increasing the total energy of the system using impulsive inputs. After increasing the energy of the system, rest-to-rest maneuvers are employed to regulate the system energy to the desired level that corresponds to the upright configuration of the acrobot. The proposed algorithm is implemented with two sets of acrobot parameters taken from the literature. As compared to the approaches in the literature, the simulation results show shorter swing-up time and lower maximum continuous torques. Furthermore, the proposed algorithm does not impose any restrictions on the initial conditions and controller gains for swing-up.
\end{abstract}

\section{NOMENCLATURE}

For the nomenclature below, $k \in\{1,2\}$ and $j \in\{1,5\}$.

$l_{k} \quad$ length of the $k$-th link $(m)$

$d_{k} \quad$ distance between the $k$-th joint and center-of-mass of the $k$-th link $(m)$

$m_{k} \quad$ mass of the $k$-th link $(k g)$

$I_{k} \quad$ mass moment of inertia of the $k$-th link about its center-of-mass $\left(\mathrm{kg} \cdot \mathrm{m}^{2}\right)$

$g \quad$ acceleration due to gravity, $\left(9.81 \mathrm{~m} . \mathrm{s}^{-2}\right)$

$\theta_{k} \quad$ angular displacement of the $k$-th link, as defined in Fig.1 ( $\mathrm{rad})$

$\dot{\theta}_{k} \quad$ angular velocity of the $k$-th link $(\mathrm{rad} / \mathrm{s})$

$\dot{\theta}_{k}^{+} \quad$ angular velocity of the $k$-th link, immediately after application of the impulsive torque $(\mathrm{rad} / \mathrm{s})$

$\dot{\theta}_{k}^{-} \quad$ angular velocity of the $k$-th link, immediately prior to application of the impulsive torque $(\mathrm{rad} / \mathrm{s})$

$\dot{\theta}_{2 d e s} \quad$ desired angular velocity of the second link after the application of the impulsive torque $(\mathrm{rad} / \mathrm{s})$

$v_{k} \quad$ velocity of the center-of-mass of the $k$-th link $(\mathrm{m} / \mathrm{s})$

${\overrightarrow{v_{k}}}^{-} \quad$ velocity of the center-of-mass of the $k$-th link, immediately prior to application of the impulsive torque $(\mathrm{m} / \mathrm{s})$

$\overrightarrow{v k}^{+} \quad$ velocity of the center-of-mass of the $k$-th link, immediately after application of the impulsive torque $(\mathrm{m} / \mathrm{s})$

$E \quad$ total energy of the acrobot $(J)$

$\vec{F}_{k}^{i m p}$ impulsive force acting at the center-of-mass of the $k$-th link $(N)$

All authors are with the Department of Mechanical Engineering, Michigan State University, East Lansing, MI, 48824, USA. The corresponding author is R. Mukherjee, email: mukherji@egr.msu.edu

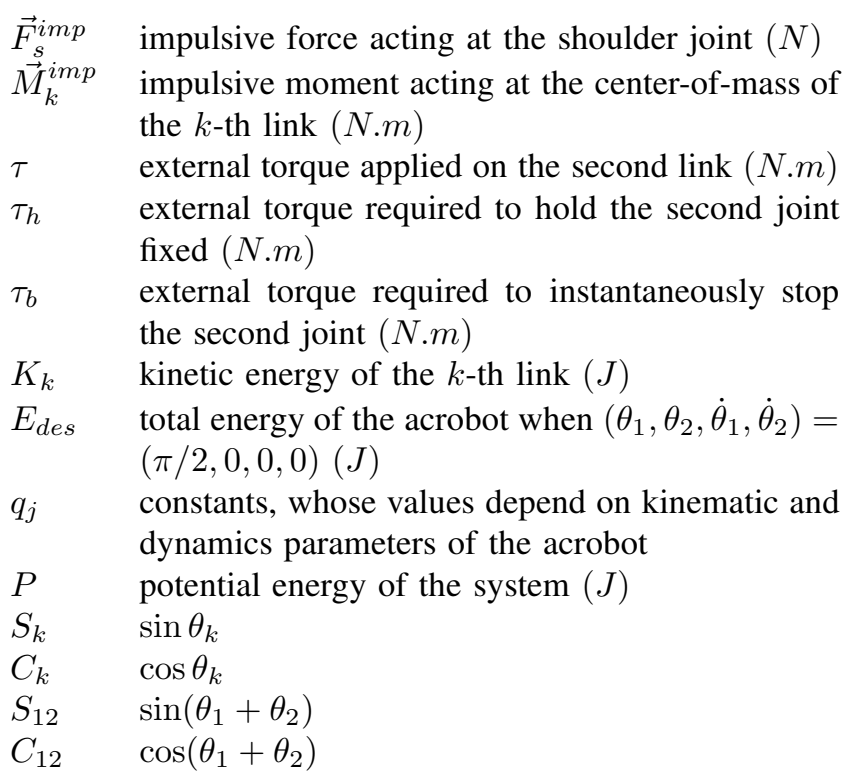

\section{INTRODUCTION}

The acrobot is a two-link robot in the vertical plane with an actuator at the elbow joint and a passive shoulder joint. It is an underactuated system and its control problem has similarities with that of the pendubot [1]. For the acrobot, the control problem requires swing-up from an arbitrary initial configuration to its configuration with maximum potential energy, followed by stabilization. The stabilization problem has seen many solutions, such as linear quadratic regulator [2], [3], and robust control [4] based designs. The swing-up control problem is more challenging and requires the system trajectory to be driven to the neighborhood of one equilibrium configuration from any point in the configuration space that contains four equilibria. Spong [2] proposed a method based on partial feedback linearization but this approach is very sensitive to the values of the control gains. Boone [5] proposed bang-bang control for near-optimal swing-up trajectories. The algorithm switches at finite time intervals and becomes computationally expensive for a large number of intervals. Xin and Kaneda [6] and Mahindrakar and Banavar [7] used a single Lyapunov function to design swingup controllers. The control design by Xin and Kaneda [6] requires an initial perturbation and a strong condition to be imposed on controller parameters to guarantee convergence. The design by Mahindrakar and Banavar [7], on the other hand, results in relatively large continuous control inputs. Lai, et al. [8] used non-smooth Lyapunov functions with fuzzy logic to remove the constraint on the control parameter in the design by Xin and Kaneda [6]. 
The main objective of this research is to explore new control methods for underactuated systems by enlarging the set of admissible control inputs to include impulsive forces. It has been pointed out [1] that conventional actuators can apply impulse-like forces and inclusion of such inputs in the set of admissible inputs can result in efficient swing-up of the pendubot [1]. The pendubot and the acrobot are benchmark problems in underactuated systems and this paper presents an impulsive control method for swing-up of the acrobot.

There has been a fair amount of theoretical research on impulsive control and credit for some of the early works goes to Pavlidis [9], Gilbert and Harasty [10], Menaldi [11] and Lakshmikantham [12]. In recent years, researchers have studied the problems of stability, controllability and observability, optimality (see [13], [14], [15], [16], for example), and diverse application problems, including underactuated systems [17], [18], [1]. In this paper, an impulse-momentum approach to swing-up control of the acrobot is proposed. The algorithm uses impulsive control inputs to increase the energy of the system. In section II we review the dynamic model of the acrobot and derive expressions for jump in velocities and change in energy due to the impulsive inputs. The change in energy of the system due to maneuvers involving impulsive inputs is studied in section III. The swing-up algorithm is proposed in section IV and numerical simulations comparing the results of our algorithm with those in the literature are presented in section V. Concluding remarks are provided in section VI.

\section{SySTEM DYNAMICS AND EFFECT OF IMPULSIVE ACTUATION}

\section{A. Equations of Motion}

Consider the acrobot in Fig.1. Assuming no friction in the joints, the equation of motion can be obtained using the Lagrangian formulation as follows [2]

$$
A(\theta) \ddot{\theta}+B(\theta, \dot{\theta}) \dot{\theta}+G(\theta)=T
$$

where

$$
\theta=\left(\begin{array}{c}
\theta_{1} \\
\theta_{2}
\end{array}\right), \quad T=\left(\begin{array}{c}
0 \\
\tau
\end{array}\right)
$$

and $A(\theta), B(\theta, \dot{\theta})$, and $G(\theta)$, given by the expressions

$$
\begin{gathered}
A(\theta)=\left[\begin{array}{cc}
q_{1}+q_{2}+2 q_{3} C_{2} & q_{2}+q_{3} C_{2} \\
q_{2}+q_{3} C_{2} & q_{2}
\end{array}\right] \\
B(\theta, \dot{\theta})=q_{3} S_{2}\left[\begin{array}{cc}
-\dot{\theta}_{2} & -\left(\dot{\theta}_{1}+\dot{\theta}_{2}\right) \\
\dot{\theta}_{1} & 0
\end{array}\right] \\
G(\theta)=g\left[\begin{array}{c}
q_{4} C_{1}+q_{5} C_{12} \\
q_{5} C_{12}
\end{array}\right]
\end{gathered}
$$

are the inertia matrix, matrix containing the Coriolis and centrifugal forces, and vector of gravity forces, respectively. In (3), (4), and (5), $q_{j}, j=1,2, \cdots, 5$ are positive constants obtained in the literature, (see [8], for example).

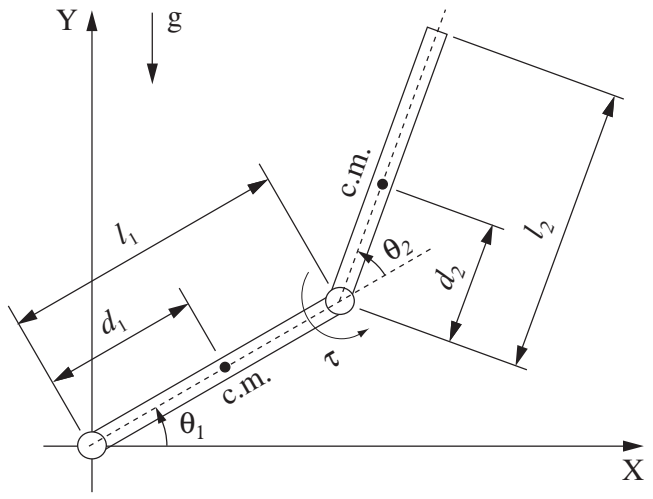

Fig. 1. The acrobot in an arbitrary configuration: the joint angles $\theta_{1}$ and $\theta_{2}$ are measured counter-clockwise with respect to the horizontal axis.

\section{B. Holding Torque}

We compute the torque required to hold the second link fixed, i.e., maintain $\dot{\theta}_{2}=0$. By substituting $\dot{\theta}_{2}=\ddot{\theta}_{2}=0$ in (1), we get

$$
\begin{aligned}
{\left[\begin{array}{c}
q_{1}+q_{2}+2 q_{3} C_{2} \\
q_{2}+q_{3} C_{2}
\end{array}\right] \ddot{\theta}_{1}+\left[\begin{array}{c}
0 \\
q_{3} S_{2} \dot{\theta}_{1}^{2}
\end{array}\right] } & +g\left[\begin{array}{c}
q_{4} C_{1}+q_{5} C_{12} \\
q_{5} C_{12}
\end{array}\right] \\
& =\left(\begin{array}{c}
0 \\
\tau_{h}
\end{array}\right)
\end{aligned}
$$

By eliminating $\ddot{\theta}_{1}$ from the two equations in (6), $\tau_{h}$ can be expressed as follows

$$
\begin{array}{r}
\tau_{h}=q_{3} S_{2} \dot{\theta}_{1}^{2}+\frac{g}{q_{1}+q_{2}+2 q_{3} C_{2}}\left[\left(q_{1}+q_{3} C_{2}\right) q_{5} C_{12}\right. \\
\left.-\left(q_{2}+q_{3} C_{2}\right) q_{4} C_{1}\right]
\end{array}
$$

\section{Impulsive Torque for Change in Velocity}

We consider the action that results in exponential convergence of the second link velocity, $\dot{\theta}_{2}$, to some desired value, $\dot{\theta}_{2 d e s}$. Therefore, we assume

$$
\ddot{\theta}_{2}=-k_{1}\left(\dot{\theta}_{2}-\dot{\theta}_{2 d e s}\right), \quad k_{1}>0
$$

where $k_{1}$ is a positive constant that will determine the rate of convergence of $\dot{\theta}_{2}$. To compute the torque required for this action, we multiply (1) with the inverse of the inertia matrix to obtain

$$
\left(\begin{array}{c}
\ddot{\theta}_{1} \\
\ddot{\theta}_{2}
\end{array}\right)=\frac{1}{q_{1} q_{2}-q_{3}^{2} C_{2}^{2}}\left[\begin{array}{c}
-\left(q_{2}+q_{3} C_{2}\right) \tau+h_{1} \\
\left(q_{1}+q_{2}+2 q_{3} C_{2}\right) \tau+h_{2}
\end{array}\right]
$$

where $h_{1}$ and $h_{2}$ are given by the expressions

$$
\begin{aligned}
h_{1}= & q_{2} q_{3}\left(\dot{\theta}_{1}+\dot{\theta}_{2}\right)^{2} S_{2}+q_{3}^{2} \dot{\theta}_{1}^{2} S_{2} C_{2} \\
& +g\left(q_{3} q_{5} C_{2} C_{12}-q_{2} q_{4} C_{1}\right) \\
h_{2}= & -\left(\dot{\theta}_{1}+\dot{\theta}_{2}\right)^{2}\left(q_{2} q_{3}+q_{3}^{2} C_{2}\right) S_{2} \\
& -\left(q_{1}+q_{3} C_{2}\right) q_{3} \dot{\theta}_{1}^{2} S_{2}-g\left\{q_{3} q_{5} C_{2} C_{12}\right. \\
& \left.-\left(q_{2}+q_{3} C_{2}\right) q_{4} C_{1}+q_{1} q_{5} C_{12}\right\}
\end{aligned}
$$

Substituting (8) into the second equation in (9) results in

$$
\tau=\frac{-1}{q_{1}+q_{2}+2 q_{3} C_{2}}\left[k_{1}\left(\dot{\theta}_{2}-\dot{\theta}_{2 d e s}\right)\left(q_{1} q_{2}-q_{3}^{2} C_{2}^{2}\right)+h_{2}\right]
$$


If the gain $k_{1}$ is chosen very large, the torque in (12) will be impulsive in nature and will converge the second link velocity to the desired velocity in a very short period of time.

As a special case, consider the impulsive action that results in stopping the second link. By setting the desired velocity of the second link in (12) to zero, i.e., $\dot{\theta}_{2 d e s}=0$, the impulsive braking torque is computed as

$$
\tau_{b}=\frac{-1}{q_{1}+q_{2}+2 q_{3} C_{2}}\left[k_{1} \dot{\theta}_{2}\left(q_{1} q_{2}-q_{3}^{2} C_{2}^{2}\right)+h_{2}\right]
$$

When the second joint comes to rest, the braking torque becomes equal to the holding torque. This can be verified from (7) and (13).

\section{Impulse-Momentum Effect: Change in Velocity}

An external impulsive torque results in impulsive forces and moments acting on both links of the acrobot. From the free-body of the second link in Fig.2(a), we can write

$$
\begin{aligned}
\vec{F}_{2}^{i m p} \Delta t & =m_{2}\left({\overrightarrow{v_{2}}}^{+}-{\overrightarrow{v_{2}}}^{-}\right) \\
\vec{M}_{2}^{i m p} \Delta t & =I_{2}\left(\dot{\theta}_{1}^{+}+\dot{\theta}_{2}^{+}\right)-I_{2}\left(\dot{\theta}_{1}^{-}+\dot{\theta}_{2}^{-}\right)
\end{aligned}
$$

where $\Delta t$ is the short interval of time over which the impulsive force and impulsive moment act, and ${\overrightarrow{v_{2}}}^{+}$and ${\overrightarrow{v_{2}}}^{-}$ are given by the expressions

$$
\begin{gathered}
{\overrightarrow{v_{2}}}^{+}=-\left[l_{1} \dot{\theta}_{1}^{+} S_{1}+d_{2}\left(\dot{\theta}_{1}^{+}+\dot{\theta}_{2}^{+}\right) S_{12}\right] \vec{i} \\
+\left[l_{1} \dot{\theta}_{1}^{+} C_{1}+d_{2}\left(\dot{\theta}_{1}^{+}+\dot{\theta}_{2}^{+}\right) C_{12}\right] \vec{j} \\
{\overrightarrow{v_{2}}}^{-}=-\left[l_{1} \dot{\theta}_{1}^{-} S_{1}+d_{2}\left(\dot{\theta}_{1}^{-}+\dot{\theta}_{2}^{-}\right) S_{12}\right] \vec{i} \\
+\left[l_{1} \dot{\theta}_{1}^{-} C_{1}+d_{2}\left(\dot{\theta}_{1}^{-}+\dot{\theta}_{2}^{-}\right) C_{12}\right] \vec{j}
\end{gathered}
$$

From the free-body diagram in Fig.2(d), we can write

$$
\begin{aligned}
\vec{F}_{1}^{i m p} \Delta t & =m_{1}\left({\overrightarrow{v_{1}}}^{+}-{\overrightarrow{v_{1}}}^{-}\right) \\
\vec{M}_{1}^{i m p} \Delta t & =I_{1}\left(\dot{\theta}_{1}^{+}-\dot{\theta}_{1}^{-}\right)
\end{aligned}
$$

where

$$
\begin{aligned}
& {\overrightarrow{v_{1}}}^{+}=d_{1} \dot{\theta}_{1}^{+}\left(-S_{1} \vec{i}+C_{1} \vec{j}\right) \\
& {\overrightarrow{v_{1}}}^{-}=d_{1} \dot{\theta}_{1}^{-}\left(-S_{1} \vec{i}+C_{1} \vec{j}\right)
\end{aligned}
$$

Using the force and moments diagrams in Figs.2(b) and (c), we can express $\vec{F}_{1}^{i m p}$ and $\vec{M}_{1}^{i m p}$ as follows

$$
\begin{aligned}
\vec{F}_{1}^{i m p} & =\vec{F}_{s}^{i m p}-\vec{F}_{2}^{i m p} \\
\vec{M}_{1}^{i m p} & =-\vec{M}_{2}^{i m p}-\left(\vec{r}_{2}-\vec{r}_{3}\right) \times \vec{F}_{2}^{i m p}-\vec{r}_{1} \times \vec{F}_{s}^{i m p}
\end{aligned}
$$

where $\vec{r}_{1}, \vec{r}_{2}$ and $\vec{r}_{3}$, shown in Fig.2, have the expressions

$$
\begin{aligned}
\vec{r}_{1} & =d_{1}\left(C_{1} \vec{i}+S_{1} \vec{j}\right) \\
\vec{r}_{2} & =d_{2}\left(C_{12} \vec{i}+S_{12} \vec{j}\right) \\
\vec{r}_{3} & =\left(d_{1}-l_{1}\right)\left(C_{1} \vec{i}+S_{1} \vec{j}\right)
\end{aligned}
$$

Substituting (21) into (18) and simplifying using (14), (15), (17), (20) and (22), we get

$$
\left[q_{1}+q_{2}+2 q_{3} C_{2}\right]\left(\dot{\theta}_{1}^{+}-\dot{\theta}_{1}^{-}\right)=\left[q_{2}+q_{3} C_{2}\right]\left(\dot{\theta}_{2}^{+}-\dot{\theta}_{2}^{-}\right)
$$

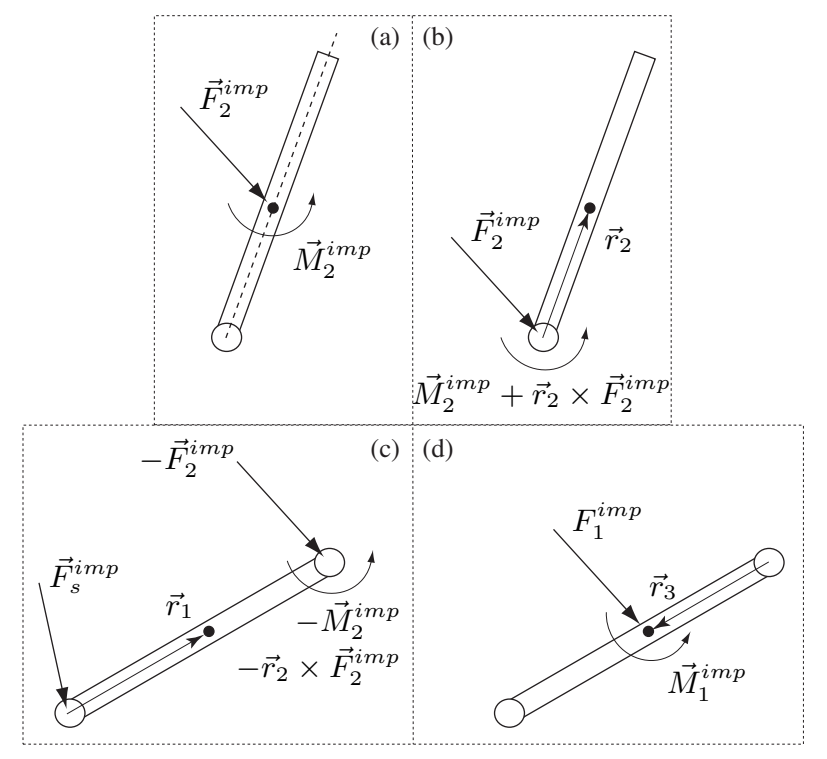

Fig. 2. Free-body diagrams showing the impulsive forces and moments acting on the second link - (a) and (b), and first link - (c) and (d), of the acrobot.

Assuming that the desired velocity of the second link after the impulse is known, i.e. $\dot{\theta}_{2}^{+}=\dot{\theta}_{2 d e s}$, (23) can be rewritten as follows to obtain expression for the velocity of the first link:

$$
\dot{\theta}_{1}^{+}=\dot{\theta}_{1}^{-}+\left[\frac{q_{2}+q_{3} C_{2}}{q_{1}+q_{2}+2 q_{3} C_{2}}\right]\left(\dot{\theta}_{2 d e s}-\dot{\theta}_{2}^{-}\right)
$$

\section{ENERGy CONSIDERATION OF THE SySTEM}

\section{A. Energy Change due to Impulsive Action}

The configuration of the acrobot will not change over the small period of time, $\Delta t$, when the impulsive torque is applied. Therefore, the change in total energy of the system is only due to the change in the kinetic energy, which can be expressed as

$$
\begin{aligned}
\Delta E= & \Delta K \\
= & \frac{1}{2} q_{1}\left[\left(\dot{\theta}_{1}^{+}\right)^{2}-\left(\dot{\theta}_{1}^{-}\right)^{2}\right]+\frac{1}{2} q_{2}\left[\left(\dot{\theta}_{2}^{+}\right)^{2}-\left(\dot{\theta}_{2}^{-}\right)^{2}\right] \\
& +q_{3} C_{2}\left[\left(\dot{\theta}_{1}^{+}+\dot{\theta}_{2}^{+}\right)-\left(\dot{\theta}_{1}^{-}+\dot{\theta}_{2}^{-}\right)\right]
\end{aligned}
$$

Assuming that the second link velocity after the impulse is equal to the desired velocity, i.e. $\dot{\theta}_{2}^{+}=\dot{\theta}_{2 d e s}$, the change in the total energy of the system can be obtained by substituting (24) into (25) as follows;

$$
\Delta E=\frac{q_{1} q_{2}-q_{3}^{2} C_{2}^{2}}{2\left(q_{1}+q_{2}+2 q_{3} C_{2}\right)}\left[\left(\dot{\theta}_{2 \text { des }}\right)^{2}-\left(\dot{\theta}_{2}^{-}\right)^{2}\right]
$$

Equation (26) implies that sign of the change in the total energy of the system depends on the magnitude of $\dot{\theta}_{2 d e s}$, i.e.

$$
\begin{array}{lll}
\Delta E>0 & \text { if } & \left|\dot{\theta}_{2 \text { des }}\right|>\left|\dot{\theta}_{2}^{-}\right| \\
\Delta E=0 & \text { if } & \left|\dot{\theta}_{2 \text { des }}\right|=\left|\dot{\theta}_{2}^{-}\right| \\
\Delta E<0 & \text { if } & \left|\dot{\theta}_{2 \text { des }}\right|<\left|\dot{\theta}_{2}^{-}\right|
\end{array}
$$


If the second link is stopped using the impulsive torque, i.e. $\dot{\theta}_{2 \text { des }}=0$, the energy loss due to the braking is obtained from (26) as:

$$
\Delta E=-\frac{q_{1} q_{2}-q_{3}^{2} C_{2}^{2}}{2\left(q_{1}+q_{2}+2 q_{3} C_{2}\right)}\left(\dot{\theta}_{2}^{-}\right)^{2}
$$

\section{B. Rest-to-Rest Maneuver of the second link}

Consider a maneuver in which the second joint starts from rest and is brought back to rest through the application of the impulsive braking torque in (13), where gain $k_{1}$ has a large value. Taking into account the loss of energy due to impulsive braking given by (28), the net work done on the system during the rest-to-rest maneuver can be computed as follows:

$$
\Delta E=\int \tau \dot{\theta}_{2} d t-\frac{q_{1} q_{2}-q_{3}^{2} C_{2}^{2}}{2\left(q_{1}+q_{2}+2 q_{3} C_{2}\right)}\left(\dot{\theta}_{2}^{-}\right)^{2}
$$

If the torque in (29) is assumed to have the form

$$
\tau=k_{2} \ddot{\theta}_{2}
$$

where $k_{2}$ is a positive constant, the work done by the torque to move the second link from rest to the velocity $\dot{\theta}_{2}^{-}$, which is the velocity prior to application of the impulsive brake, is expressed as follows:

$$
\begin{aligned}
\int \tau \dot{\theta}_{2} d t & =\int k_{2} \dot{\theta}_{2} \ddot{\theta}_{2} d t \\
& =\int_{0}^{\dot{\theta}_{2}^{-}} k_{2} \dot{\theta}_{2} d \dot{\theta}_{2}=\frac{1}{2} k_{2}\left(\dot{\theta}_{2}^{-}\right)^{2}
\end{aligned}
$$

The total change in energy due to the rest-to-rest maneuver can now be obtained by substituting (31) into (29):

$$
\Delta E=\frac{1}{2}\left[k_{2}-\frac{q_{1} q_{2}-q_{3}^{2} C_{2}^{2}}{\left(q_{1}+q_{2}+2 q_{3} C_{2}\right)}\right]\left(\dot{\theta}_{2}^{-}\right)^{2}
$$

From (32), it can be seen that the gain $k_{2}$ can be chosen to add or remove energy from the system, namely,

$$
\begin{aligned}
& k_{2}>\frac{q_{1} q_{2}-q_{3}^{2} C_{2}^{2}}{\left(q_{1}+q_{2}+2 q_{3} C_{2}\right)} \quad \Rightarrow \quad \Delta E>0 \\
& k_{2}<\frac{q_{1} q_{2}-q_{3}^{2} C_{2}^{2}}{\left(q_{1}+q_{2}+2 q_{3} C_{2}\right)} \quad \Rightarrow \quad \Delta E<0
\end{aligned}
$$

Using (9), the torque expression in (30) can be written as:

$$
\tau=\frac{k_{2} h_{2}}{q_{1} q_{2}-q_{3}^{2} C_{2}^{2}-k_{2}\left(q_{1}+q_{2}+2 q_{3} C_{2}\right)}
$$

The above expression will not have any singularity if $k_{2}$ is chosen based on (33).

\section{SWIng-UP Algorithm}

The swing-up of the acrobot requires its energy to be increased to the desired level that corresponds to the upright equilibrium configuration. We propose an algorithm where the energy of the system is increased through the application of impulsive torques. In particular, we propose to apply the impulsive torque in (12) where $\dot{\theta}_{2 d e s}$ is chosen to increase the energy of the system based on (27). If the velocity of the second link after the impulse is chosen as,

$$
\dot{\theta}_{2 \text { des }}=-\lambda \dot{\theta}_{2}^{-}, \quad \dot{\theta}_{2}^{-} \neq 0, \quad \lambda>1
$$

the second link will have a velocity greater in magnitude and opposite in direction to the velocity prior to the impulse, and the energy of the system will be higher. The change in sign of the velocity of the second link is needed to keep the second link angle bounded within some predefined range while the energy is raised to the desired level.

Most of the energy gained by the application of impulsive torques will be in the form of kinetic energy and has to be converted into potential energy for swing-up. To convert kinetic energy into potential energy, the torque is set to zero and the second joint is made free. This keeps the total energy of the system constant and kinetic energy is exchanged into potential energy. The rest-to-rest maneuvers are used to regulate the energy to the desired level. When the acrobot configuration is in the neighborhood of the desired equilibrium configuration, a linear controller is invoked to stabilize the equilibrium.

The rest-to-rest maneuvers alone can be used to increase the energy of the system, as proposed for the pendubot [1], but the time required for the acrobot is significant. Therefore, the rest-to-rest maneuvers are only used for fine tuning of the energy level.

The following 4-step algorithm describes the proposed approach for the acrobot swing-up in more details;

\section{Initialization:}

- Linearize the dynamic equations of the acrobot in (1) about the desired equilibrium $\left(\theta_{1}, \dot{\theta}_{1}, \theta_{2}, \dot{\theta}_{2}\right)=$ $(\pi / 2,0,0,0)$.

- Design a linear controller to render the desired equilibrium locally asymptotically stable. Let $R_{A}$ define the region of attraction of the desired equilibrium.

- Choose a small positive constant $\delta$, such that the system configuration lies inside $R_{A}$ when $\dot{\theta}_{1} \approx \dot{\theta}_{2} \approx 0$ and $\left|P-E_{\text {des }}\right|<\delta$.

2. Increasing the energy of the system:

- At the initial time, if $\theta_{2}=0$ and $\dot{\theta}_{2}=0$, apply the impulsive torque in (12), where $\dot{\theta}_{2 d e s}$ is chosen to be some nonzero velocity. From (27), we know this will increase the energy of the system.

- Using (35), apply the impulsive torque in (12) whenever the second link approaches a bound of the interval $[-\gamma, \gamma]$ from inside where $\gamma$ is a positive angle. Note that $\gamma$ can be always chosen such that the second link approaches the bound of the interval $[-\gamma, \gamma]$ for any initial conditions or initial velocity of the second link, $\dot{\theta}_{2 d e s}$. Applying these impulsive torques will increase the energy of the system, mainly in the form of kinetic energy. Continue this process till $E \geq E_{\text {des }}$.

- Release the system by setting $\tau=0$. This will cause the links to start swinging freely and there will be exchange between kinetic and potential energies while the total energy of the system remains the same. 
- Stop the second link using the impulsive braking torque in (13) when the potential energy of the acrobot reaches its local maxima, i.e, $\dot{P}=0, \ddot{P}<0$. This will reduce the total energy of the system according to (28) but the potential energy will remain at its local maxima.

\section{Regulation of energy to the desired level:}

- The energy of the system will increase significantly in step 2. Conduct rest-to-rest maneuvers to regulate the energy to $E_{\text {des }}$. The rest-to-rest maneuvers will be implemented with $\theta_{2}$ satisfying $-\alpha \leq \theta_{2} \leq \alpha$, where $\alpha$ is a small positive angle. If the second link is initially out of this bound, it will be brought inside the interval $[-\alpha, \alpha]$ by the first rest-to-rest maneuver. In particular, the following procedure will be adopted:

The holding torque in (7) will be applied to hold the second link fixed. For a desired motion of the second link in the positive (counter-clockwise) direction, the torque expression in (34) will be used when it is greater than $\tau_{h}$. For a desired motion in the negative (clockwise) direction, the torque expression in (34) will be used when it is less than $\tau_{h}$. Based on the current level of energy, the gain $k_{2}$ is chosen from (33) to increase or decrease the energy of the system.

- Terminate the rest-to-rest maneuvers when $(E-$ $\left.E_{d e s}\right)<\delta$ and apply the holding torque in (7) to keep the second link fixed.

\section{Stabilization:}

With $-\alpha \leq \theta_{2} \leq \alpha, \dot{\theta}_{2}=0$, and $\left(E-E_{\text {des }}\right)<\delta$, the first link will behave like a pendulum and reach its highest potential energy configuration in finite time. When the acrobot reaches its highest potential energy configuration, i.e. $\left(P-E_{\text {des }}\right)<\delta$ and $\dot{\theta}_{1}=\dot{\theta}_{2}=0$, the acrobot configuration will be inside $R_{A}$. Invoke the linear controller, designed in the first step of the algorithm, to stabilize the desired equilibrium.

In comparison to the approaches proposed by Xin and Kaneda [6] and Mahindrakar and Banavar [7], our approach does not impose any restrictions on the controller gains or initial conditions for swing-up of the acrobot.

\section{Numerical Simulation}

We compare the efficacy of our algorithm with those proposed in [6] and [7]. The kinematic and dynamic parameters of the first simulation are taken from Mahindrakar and Banavar [7]:

$$
\begin{array}{ll}
m_{1}=1.0 k g, & l_{1}=1 m, \quad d_{1}=0.5 m, \quad I_{1}=0.083 N . m^{2} \\
m_{2}=2.0 k g, & l_{2}=2 m, \quad d_{2}=1.0 m, \quad I_{2}=0.667 N . m^{2}
\end{array}
$$

The parameters of our algorithm are chosen as follows,

$$
\lambda=2.5, \quad \delta=0.1 J, \quad \gamma=70^{\circ}, \quad \alpha=5^{\circ}
$$

For the parameters given in (36), the energy of the acrobot at the upright configuration was calculated to be $E_{\text {des }}=$ 88.29 J. The simulation results plotting the joint angles, joint velocities, the total energy and the potential energy, and
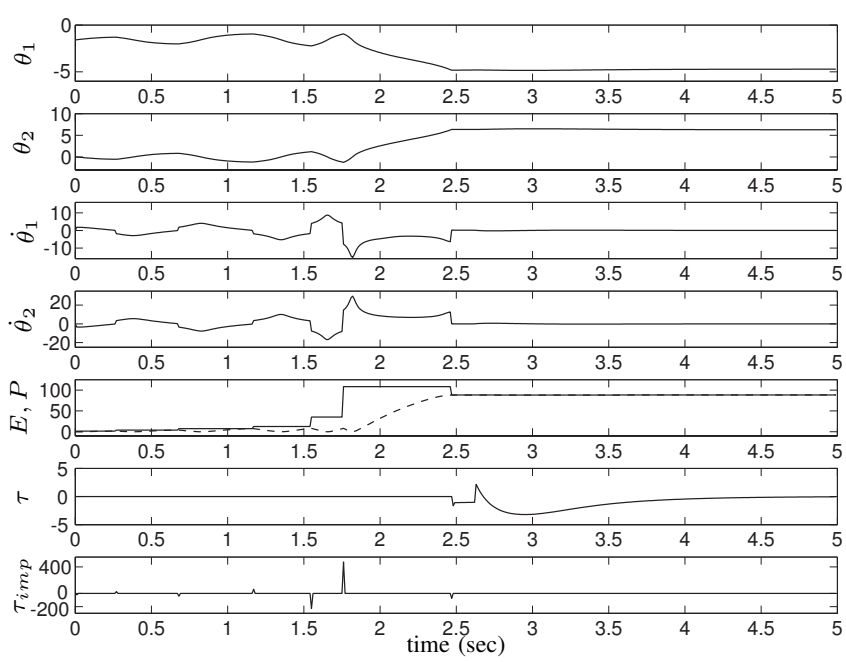

Fig. 3. Plot of joint angles (rad), joint angle velocities ( $\mathrm{rad} / \mathrm{s})$, total energy (solid line) and potential energy (dashed line) (J), continuous control input (N.m) and impulsive control input (N.m) for the first simulation

the continuous and impulsive control torques are shown in Fig.3. Since the magnitude of the continuous torque is much smaller than that of the impulsive torque, they are plotted separately such that the continuous torque can be viewed with greater resolution. The total energy (solid line) of the system increases through discrete jumps at times when the impulsive torques are applied. When the condition $E>E_{\text {des }}$ is satisfied, the second joint is released. This results in increase in the potential energy (dashed line) while the total energy remains constant. The slight drop in the energy is due to the impulsive brake when the potential energy reaches its local maxima. In this simulation, the rest-to-rest maneuvers are not required because the conditions $-\alpha \leq \theta_{2} \leq \alpha$, $\dot{\theta}_{2}=0$, and $\left(E-E_{d e s}\right)<\delta$ are all satisfied. The linear controller is invoked a short time later, at approximately $2.6 \mathrm{sec}$, when the acrobot configuration enters the region of attraction of the desired equilibrium.

The simulation results in Fig. 3 show very fast swing-up of the acrobot. The linear controller is invoked at $t=2.61 \mathrm{sec}$ as compared to 18 seconds required by the algorithm in [7]. The maximum continuous torque required by our algorithm is $3.2 \mathrm{~N} . \mathrm{m}$ which is significantly smaller than the maximum torque of 80 N.m used in [7]. Our algorithm, of course, requires impulsive torques which are large in magnitude but act over small time intervals. Some discussion on practical implementation is provided in the remark following the next simulation.

For the second simulation, we used the kinematic and dynamic parameters from Xin and Kaneda [6]:

$$
\begin{aligned}
& m_{1}=1.0 \mathrm{~kg}, \quad l_{1}=1 \mathrm{~m}, \quad d_{1}=0.5 \mathrm{~m}, \quad I_{1}=0.083 \mathrm{~N} . \mathrm{m}^{2} \\
& m_{2}=1.0 \mathrm{~kg}, \quad l_{2}=2 \mathrm{~m}, \quad d_{2}=1.0 \mathrm{~m}, \quad I_{2}=0.33 \mathrm{~N} . \mathrm{m}^{2}
\end{aligned}
$$

The parameters of our algorithm were chosen as:

$$
\lambda=2.5, \quad \delta=0.15 \mathrm{~J}, \quad \gamma=60^{\circ}, \quad \alpha=5^{\circ}
$$

For the parameters in (38), the desired level of energy was computed to be $E_{d e s}=49.05 \mathrm{~J}$. Fig. 4 shows the 


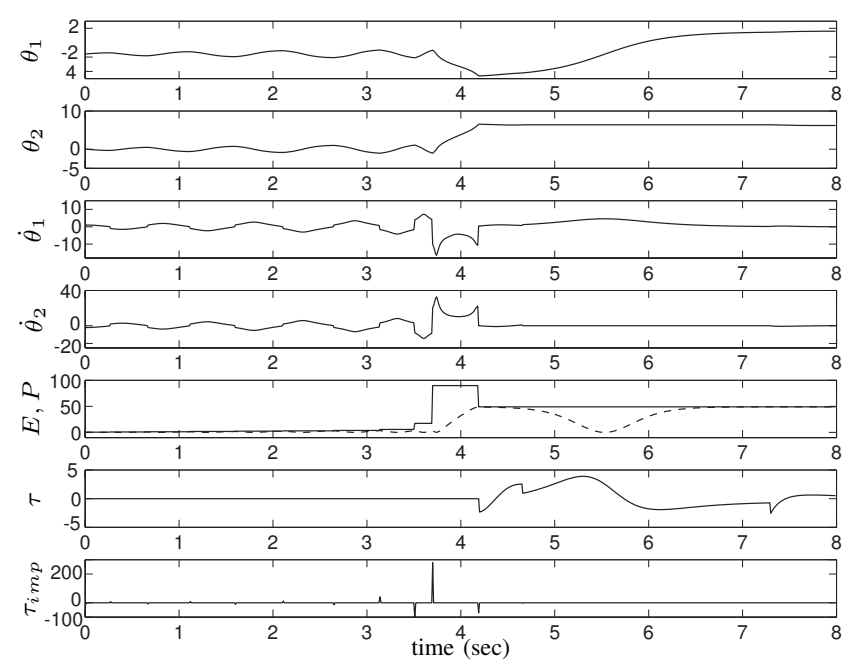

Fig. 4. Plot of joint angles (rad), joint angle velocities ( $\mathrm{rad} / \mathrm{s})$, total energy (solid line) and potential energy (dashed line) (J), continuous control input (N.m) and impulsive control input (N.m) for the second simulation

simulation results. As in the first simulation, the total energy (solid line) is discretely increased through the application of impulsive torques until $E \geq E_{\text {des }}$, which is the time the second joint is released. As the potential energy (dashed line) reaches its local maxima, the second link is stopped using an impulsive brake and this results in a drop in the total energy of the system. Unlike the first simulation, the energy after the impulsive brake does not satisfy the condition $\left|E-E_{d e s}\right|<\delta$, and therefore, the rest-to-rest maneuvers are used to regulate the energy to $E_{\text {des }}$. The linear controller is invoked at $t=7.3 \mathrm{sec}$ which is marginally better compared to 7.33 seconds required in [6]. The maximum continuous torque is $6.81 \mathrm{~N} . \mathrm{m}$ which is significantly smaller than the maximum torque, $20 \mathrm{N.m}$, used in [6].

Remark 1: In addition to continuous torques, our algorithm uses impulsive torques. Although our analysis is based on modeling the impulsive torques as Dirac delta functions, the numerical simulations indicate that the swing-up algorithm is effective even when the magnitude of the impulse is bounded and its time support is not infinitesimal. The magnitude of the impulsive inputs are however much larger than the continuous torque required by our controller, or the controllers proposed in [6] and [7]. This should not be seen as a problem since actuators such as motors can apply substantially larger torques ${ }^{1}$ than the maximum continuous torque over small time intervals.

\section{CONCLUSION}

We proposed a new algorithm for swing-up control of the acrobot. The algorithm is based on regulation of the energy of the system to some desired value, which is achieved using impulsive inputs. As the acrobot gets to its local maximum potential energy configuration, rest-to-rest maneuvers are used to regulate the system energy to the desired level. The simulation results show that our approach results in a

\footnotetext{
${ }^{1}$ This is referred to as the peak torque [19] and it can be twice to ten times larger than the maximum continuous torque for different motors.
}

faster swing-up while requiring lower maximum continuous torque as compared to those in [6] and [7]. Furthermore, our algorithm does not impose any restrictions on the initial conditions or controller gains, as is the case for the algorithm proposed in [6] and [7]. Our future work will focus on experimental verification of the impulsive control methods proposed here for swing-up of the acrobot as well as for the pendubot [1].

\section{ACKNOWLEDGMENTS}

The funding provided by the National Science Foundation, NSF Grant CMMI 0925055, is appreciated.

\section{REFERENCES}

[1] T. Albahkali, R. Mukherjee, and T. Das, "Swing-Up Control of the Pendubot: An Impulse-Momentum Approach," IEEE Transactions on Robotics, vol. 25, no. 4, pp. 975-982, 2009.

[2] M. Spong, "The swing up control problem for the acrobot," IEEE Control Systems Magazine, vol. 15, no. 1, pp. 49-55, 1995.

[3] X. Xin and M. Kaneda, "A robust control approach to the swing up control problem for the acrobot," in Proc. IEEE/RSJ Int. Conf. on Intelligent Robots and Systems, vol. 3, 2001, pp. 1650-1655.

[4] J. Leavitt, J. Bobrow, and A. Sideris, "Robust balance control of a onelegged, pneumatically-actuated, Acrobot-like hopping robot," in 2004 IEEE International Conference on Robotics and Automation, 2004. Proceedings. ICRA'04, vol. 4, 2004.

[5] G. Boone, "Minimum-time control of the acrobot," in IEEE International Conference on Robotics and Automation. Citeseer, 1997, pp. 3281-3287.

[6] X. Xin and M. Kaneda, "Analysis of the energy-based swing-up control of the Acrobot," International Journal of Robust and Nonlinear Control, vol. 17, no. 16, pp. 1503-1524, 2007.

[7] A. Mahindrakar and R. Banavar, "A swing-up of the acrobot based on a simple pendulum strategy," International Journal of Control, vol. 78, no. 6, pp. 424-429, 2005.

[8] X. Lai, M. Wu, and J. She, "Control of acrobot based on Lyapunov function," Journal of Central South University of Technology, vol. 11, no. 2, pp. 210-215, 2004.

[9] T. Pavlidis, "Stability of systems described by differential equations containing impulses," IEEE Transactions on Automatic Control, vol. 12 , no. 1, pp. 43-45, 1967.

[10] E. Gilbert and G. Harasty, "A class of fixed-time fuel-optimal impulsive control problems and an efficient algorithm for their solution," IEEE Transactions on Automatic Control, vol. 16, no. 1, 1971.

[11] J. Menaldi, "The separation principle for impulse control problems," Proceedings of the American Mathematical Society, vol. 82, no. 3, pp. 439-445, 1981.

[12] V. Lakshmikantham, D. Bainov, and P. Simeonov, Theory of impulsive differential equations. World Scientific Pub Co Inc, 1989.

[13] J. Sun, Y. Zhang, and Q. Wu, "Less conservative conditions for asymptotic stability of impulsive control systems," IEEE Transactions on automatic control, vol. 48, no. 5, p. 829, 2003.

[14] I. Stamova, Stability Analysis of Impulsive Functional Differential Equations. Walter de Gruyter, 2009.

[15] G. Xie and L. Wang, "Necessary and sufficient conditions for controllability and observability of switched impulsive control systems," IEEE Transactions on Automatic Control, vol. 49, no. 6, pp. 960-966, 2004.

[16] J. Chudoung and C. Beck, "The minimum principle for deterministic impulsive control systems," in Decision and Control, 2001. Proceedings of the 40th IEEE Conference on, vol. 4, 2001.

[17] S. Weibel, G. Howell, and J. Baillieul, "Control of single-degreeof-freedom Hamiltonian systems with impulsive inputs," in IEEE Conference on Decision and Control, 35 th, Kobe, Japan, 1996, pp. 4661-4666.

[18] Y. Aoustin, D. Romero, C. Chevallereau, and S. Aubin, "Impulsive control for a thirteen-link biped," in 9th IEEE International Workshop on Advanced Motion Control, 2006, pp. 439-444.

[19] H. Toliyat and G. Kliman, Handbook of electric motors. CRC, 2004. 